\title{
PARP-1/2-Inhibitor beim rezidivierten platinsensitiven Ovarialkarzinom
}

In einer doppelblinden Phase-IIIStudie wurden die Effizienz und Sicherheit von Niraparib als Erhaltungstherapie bei rezidiviertem platinsensitivem Ovarialkarzinom untersucht.

$\mathrm{N}$ iraparib ist ein hochselektiver Inhibitor der PARP („,poly-[ADP-ribose] polymerase") $1 / 2$, der beim rezidivierten Ovarialkarzinom in einer Phase-I-Studie zum klinischen Ansprechen bei wenig starken Nebenwirkungen führte. In der aktuellen Studie wurden 553 Patientinnen mit Ovarialtumoren von prädominant hochgradiger seröser Histologie behandelt, die sich als sensitiv gegenüber platinbasierter Chemotherapie erwiesen und mindestens zwei solcher Regimes er- halten hatten. Sie wurden je nach Anoder Abwesenheit einer KeimbahnBRCA-Mutation (gBRCA- und nongBRCA) und Typ der non-BRCA-Mutation stratifiziert und 2:1 auf Niraparib (300 mg/d) oder Placebo bis zu Krankheitsprogress oder inakzeptablen Nebenwirkungen randomisiert. Von 203 Patientinnen der gBRCA-Kohorte erhielten 138 Niraparib und 65 Placebo, in der nonBRCA-Kohorte 234 versus 116. Primärer Endpunkt war das progressionsfreie Überleben (PFS). Unter Niraparib war das PFS signifikant länger: 21,0 versus 5,5 Monate in der gBRCA-Kohorte (Hazard Ratio [HR] 0,27), 9,3 versus 3,9 Monate in der non-gBRCA-Gesamt-Kohorte (HR 0,45 ) und 12,9 versus 3,8 Monate bei den non-gBRCA-Patientinnen mit Defekten der homologen Rekombination (HRD;
HR 0,38; p < 0,001 für alle 3 Vergleiche). Auch das chemotherapiefreie Intervall und die Zeit bis zur nächsten Therapie waren nach Niraparib signifikant länger.

Fazit: Bei Patientinnen mit rezidiviertem platinsensitivem Ovarialkarzinom wurde unabhängig vom gBRCA-Mutationsoder HRD-Status ein positiver Effekt von Niraparib nachgewiesen. Unter Niraparib war das PFS signifikant länger als unter Placebo, mit längerem chemotherapiefreiem Intervall und längerer Dauer bis zur nächsten Therapie. Aussagen zum Gesamtüberleben sind derzeit noch nicht möglich.

Kathrin von Kieseritzky

Mirza MR et al. Niraparib Maintenance Therapy in Platinum-Sensitive, Recurrent Ovarian Cancer. N Engl J Med. 2016;375(22):2154-64.

\section{Ovarialkarzinom: retroperitoneale Exploration}

\section{Welchen Nutzen hat eine retroperitoneale Exploration nach optimaler Debulking-Operation. Hat sie Einfluss auf das Überleben der Patientinnen?}

$E^{\text {tim }}$ wa 10-25\% der Ovarialkarzinompatientinnen, deren Tumor primär als früh eingestuft wurde, müssen in ein FIGO-Stadium IIIC hochgestuft werden, weil sich bei der Operation befallene Lymphknoten $\left(\mathrm{N}^{+}\right)$finden. In der aktuellen Studie lag der Fokus auf Patientinnen der GOG-182-Studie mit FIGO-Stadium IIIC und keinem (R0) oder minimalem

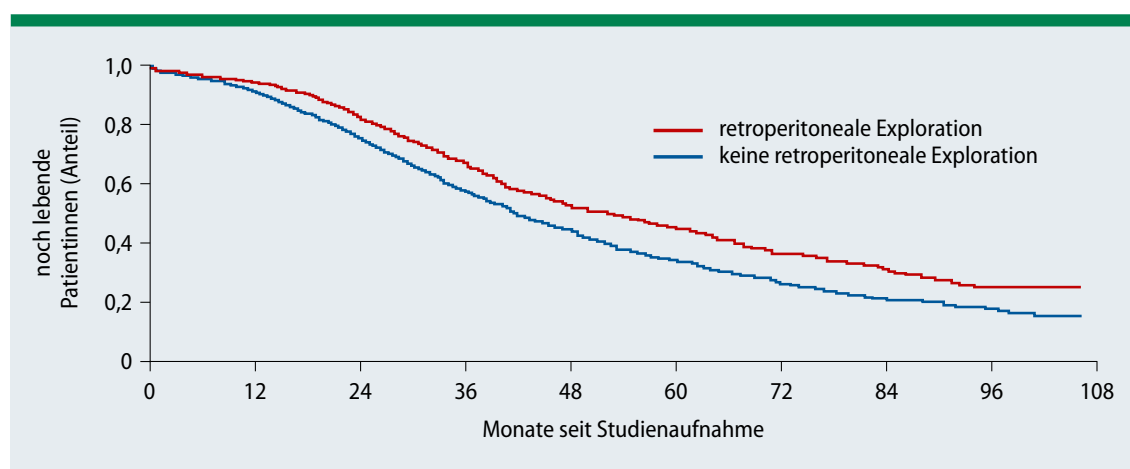

Abb. 1: Patientinnen mit minimalem Residuum $(<1 \mathrm{~cm})$, die sich einer retroperitonealen Exploration (RP) unterzogen, überlebten länger als Patientinnen ohne RP.
Residuum ( $\leq 1 \mathrm{~cm}$; MGRD; „minimal gross residual disease“) nach der zytoreduktiven Operation. Die Frauen wurden in eine Gruppe mit und in eine Gruppe ohne RP-Exploration eingeteilt. Definiert war die RP-Exploration als Entfernung von Lymphknotengewebe aus mindestens einem Bereich im Becken oder paraaortal beidseits. Postoperativ wur- 